\title{
Percepción de riesgos a la salud frente al cambio climático en México
}

\author{
Juan Alberto Gran Castro, Silvia Lizette Ramos de Robles
}

\begin{abstract}
Resumen: El objetivo de este artículo es analizar la percepción sobre riesgos a la salud y el bienestar asociados al cambio climático, a partir de las experiencias cotidianas de vivir en un contexto de marginación y pobreza urbana. La perspectiva metodológica fue de carácter cualitativo y de cohorte etnográfico, cuya principal fuente de información fueron entrevistas semiestructuradas a los padres y madres de familia, habitantes de El Colli, ubicado en Zapopan, Jalisco, México. Concluimos que las particularidades regionales a escala local enfrentan impactos específicos asociados al cambio climático según las condiciones desiguales de vulnerabilidad entre la población y la ocurrencia de eventos riesgosos. Así, sostenemos que los referentes teórico-metodológicos que consideren el conocimiento local son clave para propiciar medidas de adaptación encaminadas al bienestar social.
\end{abstract}

Palabras clave: inundaciones, pobreza urbana, urbanización, variabilidad climática, vulnerabilidad

El cambio climático ha sido considerado como el principal problema ambiental global (Delgado, 2017) y sus impactos lo han posicionado como una de las amenazas más importantes para la humanidad. Su incidencia a escala planetaria es capaz de ocasionar alteraciones en los sistemas ecológicos de soporte vital cuyas consecuencias a nivel local es necesario analizarlas desde marcos que incluyan las determinantes socioambientales, porque de ellas depende la salud humana y del ecosistema en general. En las últimas décadas, el incremento del calentamiento global de aproximadamente $1^{\circ} \mathrm{C}$ en comparación con los niveles preindustriales, ha generado alteraciones significativas de peligros meteorológicos. Las consecuencias de la alteración del sistema climático son el incremento en la probabilidad y frecuencia de eventos como olas de calor, sequías, lluvias extremas, escasez de agua, erosión de los suelos, pérdida de vegetación entre otras 
amenazas que impactan en los sistemas humanos y ambientales (Intergovernmental Panel on Climate Change, 2019).

Asimismo, la salud humana depende del funcionamiento de los sistemas ecológicos y físicos de la biósfera que fungen como un soporte vital por lo que gran parte de los eventos originados por el cambio climático traen consecuencias adversas para la salud. Aunado a esto, están los determinantes socioambientales de la salud como el acceso y calidad del agua y los alimentos. Estas situaciones han propiciado el creciente desarrollo de estudios sobre análisis de riesgos a la salud asociados al cambio climático (Watts et al., 2017). En éstos se determina que algunos de los problemas de salud más sensibles al cambio climático tienen que ver con desnutrición, salud mental, enfermedades cardiovasculares, respiratorias y transmitidas por vectores, principalmente.

En tanto, el cambio climático propicia una dinámica de multiplicidad y variabilidad de riesgos con un importante potencial de profundizar la desigualdad existente (Beck, 2010). Cuando hablamos de múltiples riesgos es indispensable señalar que, entre la ocurrencia de varias amenazas, ocurre una dinámica de sinergia y retroalimentación. Singer (2016) plantea el término pluralea, concepto compuesto del latín plur, "muchas", y alea, "amenazas". Mediante este concepto el autor busca describir la interacción entre dos o más riesgos, cuya sinergia produce impactos en el ambiente y en el bienestar de las personas. En este sentido, las poblaciones susceptibles a los impactos del cambio climático están relacionadas con un estado producido por múltiples procesos y estresores sumados al cambio climático en sí mismo (O’Brien et al., 2014).

En esta investigación nos concentramos en el estudio de los riesgos a la salud asociados al cambio climático en zonas de pobreza urbana, ya que en estos espacios existen condiciones preexistentes de vulnerabilidad e inequidad social que se suman a otros ejes de desigualdad, tanto por diferencias en la exposición a los riesgos como por los recursos disponibles para responder a los mismos. Considerando lo anterior, no todos los países ni todas las regiones o comunidades resultan afectadas de la misma manera. Para el caso de México, en el 2018 se reportaron 52.4 millones de personas en situación de pobreza y un total de 9.3 millones en pobreza extrema (CONEVAL, 2018). En el área metropolitana de Guadalajara (AMG), integrada por los municipios de Zapopan, Guadalajara, Tlaquepaque, Tonalá, El Salto, Juanacatlán, Zapotlanejo e Ixtlahuacán de los Membrillos, se concentran 1,406,834 de personas que viven en un estado de pobreza, lo cual representa aproximadamente $28.9 \%$ del total de sus habitantes y $17.7 \%$ de la población en pobreza del estado de Jalisco (CONEVAL, 2015).

Como área de estudio elegimos a la comunidad de El Colli, ubicada en el municipio de Zapopan, en el AMG, Jalisco, México. Decidimos elegir 
esta zona como área de estudio ya que El Colli pertenece a un espacio caracterizado por su abrupto crecimiento a raíz de un proceso desordenado de expansión urbana. La pobreza en esta zona alcanza un rango de pobreza medio ${ }^{1}$ para $36.8 \%$ de este espacio, alto $26.3 \%$ y muy alto $8.8 \%$ (CONEVAL, 2015). Al mismo tiempo, El Colli ha sido catalogado como una zona de alto riesgo a inundaciones e incendios forestales, cuyos pronósticos apuntan a un aumento en frecuencia e intensidad debido al cambio climático (Gran \& Ramos, 2019). Por tanto, la importancia de la percepción subjetiva del riesgo estriba en ser un factor determinante en las relaciones y comportamientos tanto individuales como familiares y grupales ante la emergencia, así como el manejo a mediano y largo plazo de la amenaza (Chávez-Rodríguez 2016). Las respuestas individuales y colectivas, incluidas las que tienen que ver con la salud, son en buena parte moldeadas por la percepción del riesgo.

En este contexto, el trabajo que realizamos en El Colli se orientó por la siguiente pregunta de investigación: ¿cuál es la percepción del riesgo a la salud y el bienestar frente al cambio climático en un contexto de pobreza urbana? Para responder a la pregunta y cumplir con el objetivo de investigación, que consistió en analizar la percepción sobre riesgos a la salud y el bienestar asociados al cambio climático a partir de las experiencias cotidianas de vivir en un contexto de marginación y pobreza urbana, optamos por una perspectiva metodológica cualitativa de corte interpretativo. Esto fue debido a que el estudio de las percepciones implica identificar las experiencias y los significados construidos por los integrantes de dicha comunidad sobre el tema de vulnerabilidad al cambio climático. Desarrollamos esta investigación a través del levantamiento de 27 entrevistas semi-estructuradas a profundidad durante el verano de 2017, las cuales estuvieron dirigidas a jefes de familia y aplicadas por hogar, procurando que cada uno de los entrevistados habitaran en una zona de alto a muy alto índice de marginación y con al menos quince años de vivir ahí. Tomamos los quince años de habitar en el lugar como criterio de inclusión reconociendo que, al tratarse de cambio climático, es importante considerar al menos un periodo de diez a treinta años para captar las características de variabilidad y cambio que pueden variar según el elemento climático como la temperatura y la precipitación (OMM, 2011). Si bien esto es una referencia para estimación de parámetros estadísticos, hemos considerado que, para documentar las percepciones, un periodo aproximado de quince años puede ser un lapso pertinente para que las personas observen y recuerden los cambios en el comportamiento del clima que han experimentado a partir de la cotidianeidad y de sus vivencias.

La estructura del artículo es la siguiente: después de esta introducción, en el primer aparatado planteamos el alcance del estudio de percep- 
ción del riesgo. En el segundo describimos el área de estudio en donde se llevó a cabo la investigación. El tercer apartado contiene el diseño y proceso metodológico que orientó la recolección y análisis de información. Posteriormente, el cuarto apartado lo destinamos a los resultados de investigación, los cuales se basan en las narrativas y experiencias de la población en torno a los riesgos percibidos en su salud y con relación al peligro de inundación. En el quinto apartado intentamos aportar una discusión orientada por los principales hallazgos del estudio en contraste con otras aportaciones e ideas similares. Por último, en el sexto apartado, postulamos las reflexiones finales a modo de conclusión.

\section{Percepción del riesgo al cambio climático}

Este artículo planteamos que las personas que viven bajo condición de pobreza son las más susceptibles de sufrir daños relacionados con el cambio climático (Hallegatte et al., 2015). Adicionalmente, los impactos del cambio climático se profundizan en las zonas urbanas, especialmente de pobreza urbana. Sin embargo, cabe aclarar que la pobreza y la vulnerabilidad no son sinónimos: la vulnerabilidad refiere a riesgos, crisis y tensiones a las que individuos, unidades domésticas o una comunidad podrían estar sujetas y a la indefensión, es decir, la falta de medios para enfrentar una contingencia. Por otra parte, entendemos la pobreza como un factor con la capacidad de incidir en situaciones de riesgo (risk driver), pues la población más pobre tiene mayor probabilidad de verse expuesta a cierta amenaza y, por tanto, ser más vulnerable (García, 2018).

Estudiar la percepción del riesgo permite comprender los procesos sociales e individuales de interpretar una situación potencialmente perjudicial para sí mismos, su salud o la vida de otros. García (2005) explica que, en términos de percepción, el riesgo es una construcción colectiva y cultural, al igual que la aceptación al mismo. En esta perspectiva, el riesgo es una construcción intelectual de los miembros de la sociedad para realizar evaluaciones sociales de probabilidades y valores. Afirma que la percepción del riesgo es un proceso social y en sí misma una construcción cultural: "entender la percepción del riesgo implica reconocer y aceptar la dimensión social del riesgo, pues su percepción es en sí un fenómeno social y no individual. De ahí que constituya, como tal, una construcción social del riesgo que surge de acuerdo con el tipo de sociedad de la que emana, de sus creencias y visiones dominantes" (García, 2005, p. 16). Podríamos decir que las bases del riesgo como proceso de construcción social se ha desarrollado en buena parte en la investigación sobre desastres (Oliver-Smith et al., 2017; Sun y Faas, 2018). 
En términos de la relación entre percepción del riesgo y salud, los riesgos a la salud se asocian directamente con las decisiones deliberadas de las personas, en su búsqueda por equilibrar su salud y otras preocupaciones, de tal modo, decisiones que protejan la salud requieren comprender los riesgos (Ferrer \& Klein, 2015; Gaube et al., 2019). Como mencionamos anteriormente, la percepción del riesgo a la salud se puede definir como la evaluación subjetiva de la probabilidad respecto a la ocurrencia de un tipo específico de accidente y la preocupación respecto a las consecuencias (Liu et al., 2013). En tanto, el riesgo percibido no se encuentra tan sólo en función de la probabilidad estimada del daño, sino que existen una serie de factores que lo integran, tales como la experiencia y la información, así como el contexto sociocultural y político de las personas.

En dicho tenor, Luhmann (2006) explica que el riesgo no depende de las características objetivas que derivan de los daños sino del modo en que éste es observado. Por lo tanto, determina que el riesgo podría concebirse como mecanismo de observación, es decir, que los eventos del mundo serán riesgosos hasta ser vistos desde la óptica del riesgo y, por tanto, pensar en el daño futuro se encuentra en función de la decisión presente. El problema es, por tanto, que los riesgos serán reales para la sociedad solo si logran ser definidos como tales, pero también escenificados exitosamente: la definición y escenificación del riesgo se llevan a cabo por los medios de comunicación y dependen de las relaciones de poder entre expertos (funcionarios públicos, científicos, técnicos, etc.) y legos (no expertos) (Beck, 1998).

Dicho esto, Lezama (2012) determina que los problemas ambientales derivan de una valoración social y no tan solo de su construcción física. En tanto, la conciencia y protesta sobre lo ambiental no está en función del daño infringido a la naturaleza y de las consecuencias en la calidad de vida y salud de los seres humanos, sino que depende de factores sociales y culturales que da lugar a una construcción y selección de los riesgos y problemas ambientales. Específicamente los estudios de percepción del riesgo en salud respecto al cambio climático permiten obtener información en torno a las preocupaciones, pensamientos, posturas, inquietudes y experiencias relacionadas con dicha percepción en diversas poblaciones. Lo anterior permite identificar las necesidades más relevantes para proteger la salud en contextos particulares de aplicación. Este punto lo sostienen investigaciones en la materia (Akerlof et al., 2010, 2015; Chávez-Rodríguez, 2016).

\section{Área de estudio}

El área de estudio para esta investigación está compuesta por asentamientos humanos en condiciones de marginación urbana en torno al bosque 
La Primavera y el cerro de El Colli, en el municipio de Zapopan, Jalisco. Específicamente la investigación se desarrolló en las colonias de Lomas de la Primavera, Arenales Tapatíos y Miramar. Estos asentamientos surgen a principios de los noventa y forman parte de un tipo de crecimiento urbano muy peculiar del AMG. Por ejemplo, De la Peña (2000) explica que a partir de 1965 la tierra privada de la ciudad se agotó casi en su totalidad, por lo cual comenzaron a proliferar los asentamientos irregulares hacia la periferia de la ciudad en tierra ejidal. Posteriormente la creación de la Comisión para la Regularización de la Tenencia de la Tierra (CORETT) en 1973 y la Ley de Asentamientos Humanos de 1976, permitieron a los "irregulares" solicitar servicios urbanos en sus espacios de ocupación. Soria (1991), Soria (1991, citado en De la Peña, 2000) nos dice que, para 1988, más de ochocientos mil personas vivían en zonas irregulares del AMG, cubriendo cinco mil hectáreas, es decir, $20 \%$ de la extensión territorial de la ciudad en aquel entonces.

Los asentamientos del área de estudio son parte del ejido Santa Ana Tepetitlán, el cual concentra $44.5 \%$ de la superficie urbanizada del bosque La Primavera (Del Castillo, 2018). El motivo del cambio de uso de suelo en

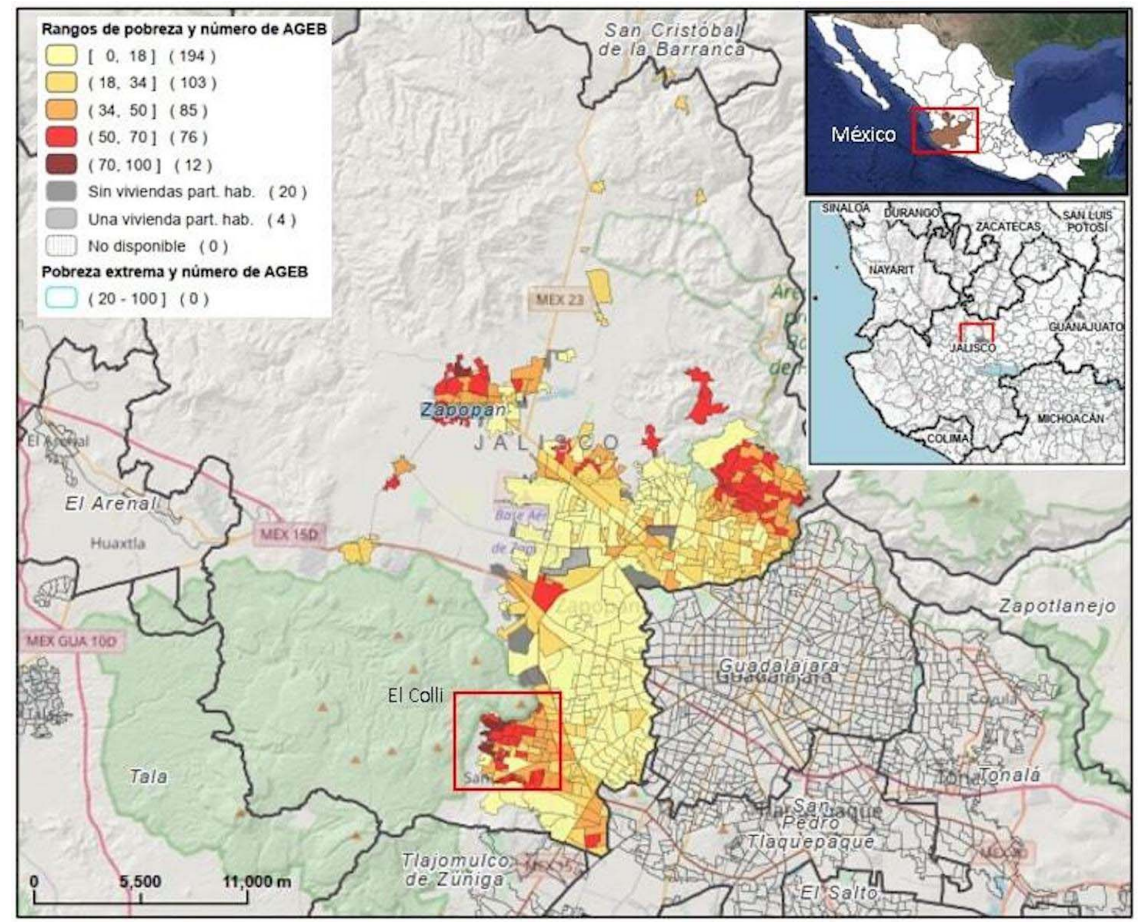

Mapa 1 - Área de estudio: El Colli, Zapopan

Fuente: elaboración propia con base en CONEVAL (2015). 
el bosque se debe al crecimiento urbano del AMG ha seguido rutas específicas de crecimiento "bordeando" ciertos límites naturales: la barranca de Huentitán, el bosque La Primavera, el valle de Toluquilla y la cuenca de El Ahogado. La principal ruta de crecimiento en términos de extensión territorial ocurre hacia los municipios de Zapopan al noroeste (con dirección al valle de Tesistán) y Tlajomulco hacia el sur-poniente, invadiendo el bosque La Primavera. Esto implica que los asentamientos en torno al bosque, tales como las colonias de Lomas de la Primavera, Arenales Tapatíos y Miramar, se ubiquen históricamente como una zona de alto riesgo a inundaciones, pues algunas de sus viviendas fueron construidas en lo que anteriormente eran arroyos y escorrentías del bosque.

Lo que constituye actualmente el contexto del área de estudio podría considerarse como una interfaz periurbana altamente segregada y heterogénea. En las colonias de Arenales Tapatíos y Miramar se aprecian contrastes importantes en términos de calidad de la vivienda y el acceso a servicios básicos: por un lado, existen calles sin pavimentación, alumbrado ni alcantarillado, con viviendas constituidas por materiales precarios; por el otro, algunas tienen todos estos servicios y otros más, como rutas de transporte público cercanas y un par de parques públicos. A su vez, conforme avanzamos hacia las zonas más lejanas de la perife-

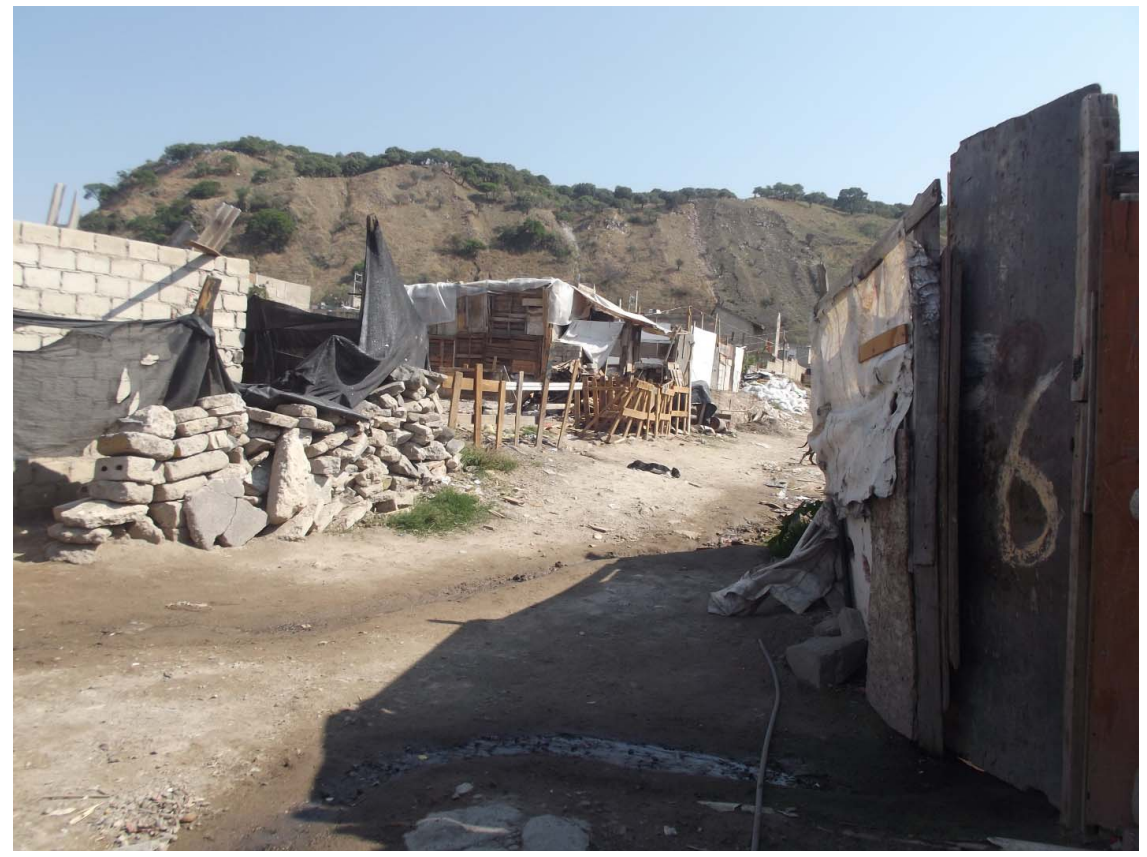

Fotografía 1 - Viviendas en las cercanías del cerro El Colli

Fuente: propia. 
ria urbana acercándose al bosque está la colonia Lomas de la Primavera, en donde las casas van cobrando características de mayor precariedad al conformarse de materiales improvisados como palos, lonas, trozos de madera y láminas. De igual modo, el entorno urbano carece de los servicios básicos.

\section{Métodos}

Nuestro objeto de investigación en este artículo es analizar la percepción sobre riesgos a la salud y el bienestar asociados al cambio climático a partir de las experiencias cotidianas de vivir en un contexto de marginación y pobreza urbana, como lo es el área de El Colli, Zapopan. Previamente, en Gran y Ramos (2019) publicamos un estudio sobre la vulnerabilidad que enfrenta la población de El Colli frente al cambio climático, en el cual integramos diversas herramientas metodológicas para el análisis, incluyendo un acercamiento de percepción del riesgo. Ahora, en este artículo, nos gustaría profundizar y centrarnos específicamente en los logros que obtuvimos respecto a la percepción de las personas.

Para el cumplimiento de nuestro objetivo de investigación seleccionamos un abordaje desde la perspectiva cualitativa de alcance descriptivo. El principal instrumento de recolección de datos fue la entrevista semiestructurada, dirigida a los jefes de familia de El Colli y aplicada por hogar. Para el desarrollo de estas solicitamos el consentimiento previo e informado de cada uno de los participantes, explicando las intenciones del estudio e invitando a tener una participación libre para responder la entrevista. Aplicamos las entrevistas entre los meses de enero y octubre de 2017, correspondientes al periodo disponible para la elaboración del proyecto de investigación. La aplicación se llevó a cabo en zonas identificadas entre un rango de pobreza medio a muy alto según la clasificación de CONEVAL (2015) por área geoestadística básica (AGEB)² (véase mapa 2 ), entre los meses de enero y octubre del 2017. La delimitación de este periodo estuvo pautada metodológicamente a través de la estrategia de "bola de nieve" y hasta llegar al punto de saturación. La estrategia de "bola de nieve", corresponde a una técnica de muestreo secuencial en la cual la integración de la muestra se define sobre la marcha a partir de las sugerencias de los propios entrevistados. En total realizamos una muestra de 27 entrevistados con los cuales se alcanzó el punto de saturación en torno a los temas de la entrevista. El punto de saturación se logra cuando el investigador ha obtenido la información suficiente respecto a todas las dimensiones de interés y/o los temas y que una nueva entrevista aportaría poca información nueva o bien, información de poca relevancia. 


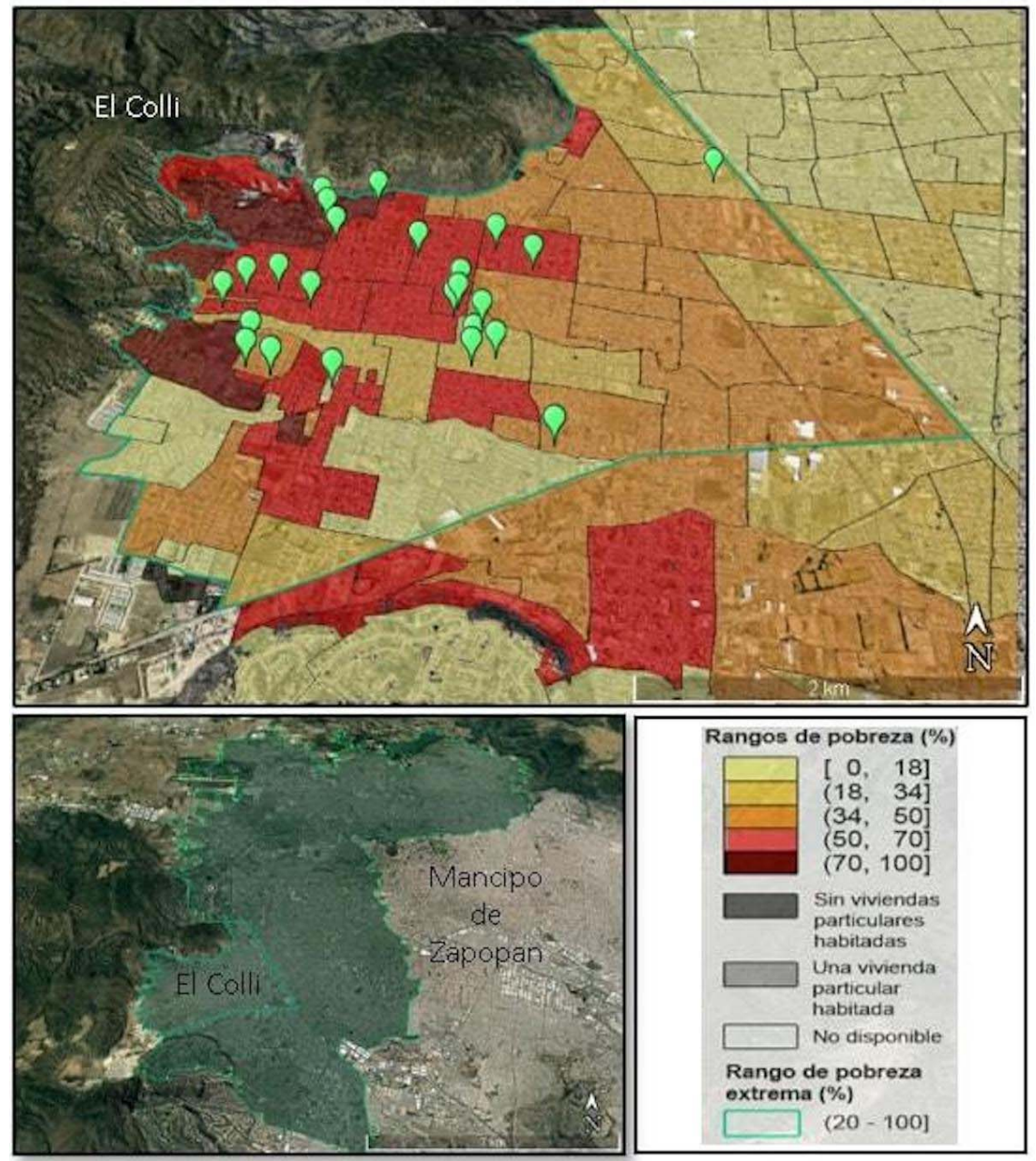

Mapa 2 - Puntos del levantamiento de entrevistas en el área de estudio, El Colli, Zapopan

Fuente: elaboración propia con base en datos de entrevistas y CONEVAL (2015).

Decidimos que los participantes fueran el padre o la madre de la familia con la intención de capturar la percepción desde la esfera individual, familiar y comunitaria y, al mismo tiempo, recuperar experiencias de personas con mayor tiempo de haber vivido en la comunidad.

El diseño de la entrevista lo realizamos a partir de la revisión de investigaciones que también abordaron la percepción del riesgo a la salud por cambio climático (principalmente Akerlof et al., 2010, 2015). Con un primer borrador, la entrevista fue validada a partir de la revisión de tres investigadores expertos en el tema y a través de una prueba piloto, cuya 
muestra estuvo integrada por cinco habitantes de El Colli. El formato final de la entrevista contempló un total de 39 preguntas que abordaron dos grandes temas: la percepción de las amenazas a la salud por cambio climático que integra la descripción de las condiciones climáticas y sus cambios, identificación de la peligrosidad y exposición al cambio climático, efecto en los grupos más afectados por peligros climáticos. Y la percepción de la vulnerabilidad frente al cambio climático que se compone de experiencias relacionadas con las amenazas más frecuentes en la comunidad, como las inundaciones según señalaron los resultados $y$, finalmente, confianza en las instituciones y apoyo; al abordar estos puntos, el peligro ante inundaciones surgió como un tema emergente entre los entrevistados, volviéndose en una de las líneas centrales para este trabajo. La entrevista también integró preguntas sobre el contexto en el que vive el entrevistado y sobre características sociodemográficas.

A la par de las entrevistas, realizamos recorridos de campo en las colonias de El Colli, en los cuales tomamos datos a través de registros de observación y fotografías, lo cual permitió contrastar y enriquecer los testimonios de los entrevistados. Un par de recorridos fueron guiados por dos actores clave de la zona: un líder religioso y una ciudadana presidenta de colonos.

Lo primero que realizamos para llevar a cabo el análisis de datos fue la trascripción de las entrevistas. Para el análisis de datos, recurrimos al método de análisis de contenido cualitativo el cual, de acuerdo con López (2002), se sitúa en el ámbito de la investigación descriptiva y pretende descubrir los componentes y significados de una situación determinada para extraerlos de un contenido dado a través de un proceso caracterizado por el intento de rigor de medición. El análisis de contenido cualitativo es un método para analizar datos e interpretar su significado. La investigación que utiliza el análisis de contenido cualitativo se centra en las características del lenguaje como comunicación con atención al contenido o al significado contextual del texto. Los datos de texto pueden estar en forma verbal, impresa o electrónica y pueden haber sido obtenidos a partir de respuestas narrativas, preguntas de encuestas abiertas, entrevistas, grupos focales, observaciones o medios impresos como artículos, libros o manuales.

Por lo tanto, todo lo dicho y escrito es susceptible de ser sometido a un análisis de contenido, herramienta útil para comprender los significados del contenido con relación al contexto de la investigación y los participantes. A partir de estos planteamientos analizamos los textos narrativos para la identificación de unidades de significado que provienen de "la autointerpretación que los sujetos relatan en primera persona, donde la dimensión temporal y biográfica ocupa una posición central" (Bolívar, 2002, p. 4). Asignamos una "etiqueta" a cada unidad de significado para representar el discurso de los participantes. 
Tabla 1 - Perfil sociodemográfico de los entrevistados

\begin{tabular}{|c|c|c|}
\hline & Suma & Porcentaje \\
\hline \multicolumn{3}{|l|}{$\overline{\text { Sexo }}$} \\
\hline Hombres & 5 & 19 \\
\hline Mujeres & 22 & 81 \\
\hline Total & 27 & 100 \\
\hline \multicolumn{3}{|l|}{ Edad } \\
\hline 15 a 24 años & 1 & 4 \\
\hline 25 a 34 años & 5 & 19 \\
\hline 35 a 44 años & 5 & 19 \\
\hline 45 a 64 años & 10 & 37 \\
\hline 65 y más años & 3 & 11 \\
\hline Sin respuesta & 3 & 11 \\
\hline \multicolumn{3}{|l|}{ Estado Civil } \\
\hline Casada(o) & 25 & 92 \\
\hline Unión libre & 1 & 4 \\
\hline Sin respuesta & 1 & 4 \\
\hline \multicolumn{3}{|c|}{ Nivel de estudios concluidos } \\
\hline Primaria & 6 & 22 \\
\hline Secundaria & 12 & 44 \\
\hline Preparatoria & 3 & 11 \\
\hline Universidad & 3 & 11 \\
\hline Sin estudios & 2 & 7 \\
\hline Sin respuesta & 1 & 4 \\
\hline \multicolumn{3}{|l|}{ Ocupación } \\
\hline Comercio propio & 5 & 19 \\
\hline Empleada(o) & 1 & 4 \\
\hline Empleada y Hogar & 12 & 44 \\
\hline Jubilado & 1 & 4 \\
\hline Sin respuesta & 1 & 4 \\
\hline \multicolumn{3}{|c|}{ Ingresos mensuales percibido por hogar } \\
\hline De 1 a 3 mil & 15 & 56 \\
\hline De 4 a 6 mil & 8 & 30 \\
\hline De 7 a 9 mil & 2 & 7 \\
\hline De 10 a 12 mil & 1 & 4 \\
\hline Sin respuesta & 1 & 4 \\
\hline \multicolumn{3}{|c|}{ Afiliación a servicios de salud } \\
\hline Seguro popular & 17 & 63 \\
\hline IMSS & 2 & 7 \\
\hline IMSS y Privado & 1 & 4 \\
\hline Privado & 1 & 4 \\
\hline Ninguno & 5 & 19 \\
\hline Sin respuesta & 1 & 4 \\
\hline
\end{tabular}

Fuente: elaboración propia con base a resultados de entrevistas. 
Este análisis lo desarrollamos con apoyo del software MAXQDA Analytics Pro-12 a partir del siguiente proceso: (1) ubicación de unidades de significado; (2) codificación de fragmentos narrativos; (3) redacción de memos; (4) revisión-validación de unidades de significado; (5) elaboración de esquemas matriciales; (6) organización de fragmentos narrativos; y (7) presentación de resultados utilizando fragmentos narrativos como evidencia. Finalmente, para presentar las expresiones directas de los entrevistados optamos por referirles como "Entrevistado 1", "2", "3", etcétera, con la finalidad de mantener el anonimato.

\section{Resultados}

Los textos narrativos, producto de las entrevistas, nos permitieron estructurar la presentación de resultados siguiendo una estructura lógica que va describiendo las percepciones de forma secuencial. Iniciamos con las vivencias en torno a las formas en que llegaron a habitar en la comunidad, para posteriormente analizar los cambios percibidos en el comportamiento del clima, los problemas de salud en relación con el temporal (calor, frío y lluvias) y, finalmente, las experiencias en torno los peligros identificados en la comunidad, específicamente aquellos asociados a las inundaciones.

\section{Asentamientos irregulares: oportunidades y desilusiones}

Los entrevistados comentan que el espacio en donde habitan comenzó a construirse a finales de los ochenta y principios de los noventa. En esa época se integraba por unas cuantas casas, siendo un espacio de muy baja densidad poblacional con uso principalmente agrícola. Expresan que el crecimiento urbano fue sorpresivo pues, en pocos años, surgieron diversas colonias de manera inesperada. El motivo de inmersión al territorio fue, en esencia, hacerse de una vivienda propia a bajo costo (o incluso gratis) ya que los precios para adquirir un terreno en El Colli eran bastante bajos en comparación con otras zonas dentro de la periferia de la ciudad. Los entrevistados coinciden en que El Colli ha sido un territorio con un proceso de crecimiento desordenado. Este cambio ha traído pocos beneficios pues las personas dicen preferir las cosas como eran antes. En general existe insatisfacción debido a la escasez de servicios públicos como agua entubada, alumbrado público y pavimento de calles:

En término de cinco años se invadió. Se llenó. Hasta yo decía, ahí por los quince años va a estar (guarda un breve silencio). Fue antes de los 
quince años. Allá del lado del cerro se empezó a fincar. Ahorita ya están más arriba. Ahora, de este lado, hace como unos cinco años, invadieron. (Entrevistado 3, junio 18, 2017)

Se puso el teléfono, se puso la luz, pusieron el drenaje, pero ya (refiriéndose solo a su colonia). De un tiempo para acá, ya nada, ¡nada en esta colonia! Parece que no existimos. Aquí no sirve nada ya. (Entrevistada 8, junio 3, 2017)

En este caso, la necesidad y urgencia de tener un terreno para autoconstruir una casa, ha impedido a los pobladores analizar las consecuencias, o bien, analizan y aceptan porque no existen más opciones plausibles. Es decir, reconocen que el crecimiento fue desordenado y que faltan servicios públicos, están insatisfechos de vivir ahí, pero esa era la mejor opción en su momento y quizá sigue siendo.

\section{Percepción sobre el cambio en el "comportamiento" del clima}

Los entrevistados, quienes han vivido por 15 años o más en la comunidad, perciben cambios en el comportamiento del clima. Tomando un periodo

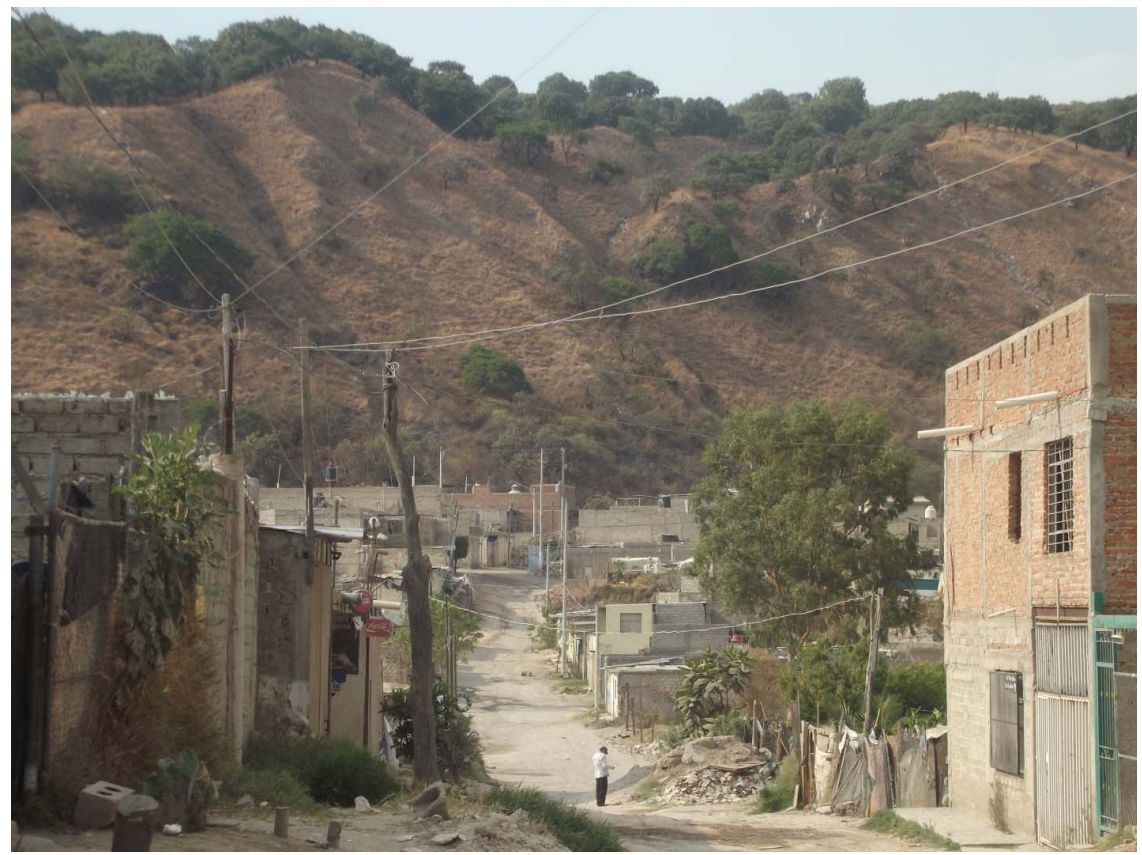

Fotografía 2 - Colonia Lomas de La Primavera con vistas al cerro de El Colli, Zapopan

Fuente: propia. 
Tabla 2 - Percepción de la comunidad de El Colli respecto a los cambios en el comportamiento del clima en los últimos quince años

\begin{tabular}{|c|c|c|c|}
\hline \multirow[b]{2}{*}{ Meses del año } & \multirow[b]{2}{*}{ Temporal } & \multicolumn{2}{|c|}{ Cambios } \\
\hline & & Incremento & Disminución \\
\hline Enero & \multirow[t]{2}{*}{ Frío } & \multirow[t]{2}{*}{ Días con frío intenso } & \\
\hline Febrero & & & \\
\hline Marzo & \multirow[t]{4}{*}{ Calor } & \multirow[t]{4}{*}{ Días con calor intenso } & \\
\hline Abril & & & \\
\hline Mayo & & & \\
\hline Junio & & & \\
\hline Julio & \multirow[t]{4}{*}{ Lluvia } & \multirow{4}{*}{$\begin{array}{l}\text { Días con lluvia intensa } \\
\text { Días calurosos }\end{array}$} & \multirow[t]{4}{*}{ Días con lluvia } \\
\hline Agosto & & & \\
\hline Septiembre & & & \\
\hline Octubre & & & \\
\hline Noviembre & \multirow[t]{2}{*}{ Frío } & \multirow[t]{2}{*}{ Días calurosos } & \multirow[t]{2}{*}{ Días con frío } \\
\hline Diciembre & & & \\
\hline
\end{tabular}

Fuente: elaboración propia con base en entrevistas realizadas en campo.

de referencia de aproximadamente quince años, mencionaron que existen alteraciones importantes asociadas con incremento del número de días calurosos y disminución de días fríos en los meses de noviembre y diciembre, así como incremento de días con frío intenso en enero y febrero; incremento de días con calor intenso durante el temporal de frío entre los meses de marzo a junio; incremento de días calurosos y días con lluvia intensa y disminución de días con lluvia.

En general, señalan un incremento de la temperatura durante la mayor parte del año; también hubo una afirmación clara sobre la variabilidad climática en donde las temperaturas de un día común resultan impredecibles, lo cual genera problemas de salud:

Mira, yo me acuerdo antes, pues llovía, como que ya tenía su temporada, por ejemplo, el 14 de mayo, mi abuelita, yo me acuerdo que nos decía, los doce primeros del año te van a decir cómo va a estar el año y pasaba lo que mi abuelita decía. Ahorita ya no, es imposible. Años anteriores sabías más o menos lo que iba a pasar, ahorita ya no. Mi papá es médico y tenemos una farmacia ahí en mi casa que es tu casa. Yo me acuerdo que antes llegaban el frío y llegaban todos enfermos, llegaba el tiempo de calor y llegaban una-dos personas. Ahorita todo el tiempo hay enfermos, todo el tiempo hay gripas, todo el tiempo hay tos, ya es totalmente variable todo. (Entrevistada 12, agosto 8, 2017) 


\section{Percepciones sobre los problemas de salud}

Tras considerar los cambios en el comportamiento del clima percibidos durante los últimos quince años, los entrevistados expresaron una serie de enfermedades que resienten como consecuencia de esto y en relación con los temporales de calor, frío y lluvia (figura 1). En el temporal de calor destacan los dolores de cabeza, enseguida las enfermedades diarreicas, estrés, asma en niños, quemaduras en la piel, influencia en la presión arterial alta, golpes de calor y finalmente afectaciones en la piel no identificadas que los entrevistados describieron como pequeños granos o erupciones. En relación con el temporal de frío hubo pocas referencias a problemas de salud, al igual que durante el temporal de lluvias. En contraste con la tabla 2, es importante señalar que los entrevistados indican padecer más problemas de salud asociados a las temperaturas altas y, al mismo tiempo, perciben un cambio en el comportamiento de las temperaturas con un incremento de días calurosos durante el año.

La explicación de algunos entrevistados frente a la variación del clima se inclinó mayoritariamente a la contaminación del aire. También existieron explicaciones con referentes bíblicos que han anunciado que el mundo se acabará en fuego. La situación sobre el comportamiento del clima genera preocupación entre los participantes sobre el bienestar familiar y comunitario, pero también generacional al pensar en sus hijos y nietos. Las acciones más típicas que los entrevistados practican son cuidar la ali-

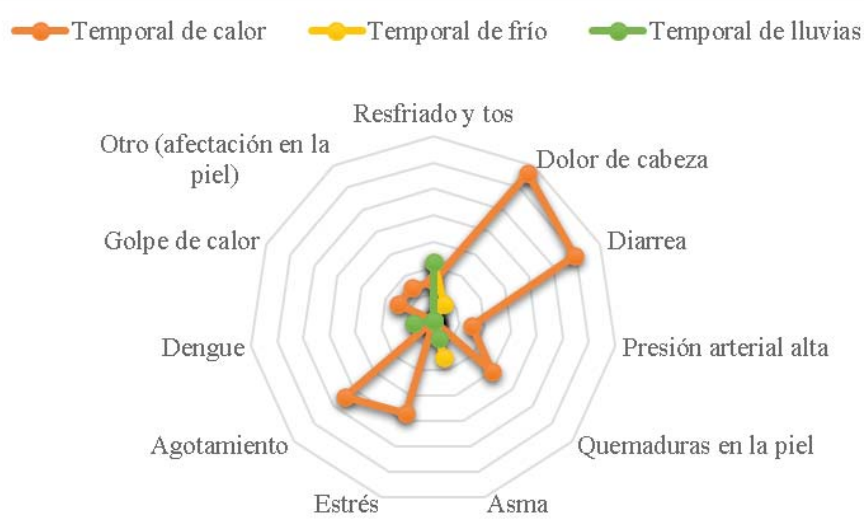

Figura 1 - Percepción en la comunidad de El Colli acerca de la relación de los problemas de salud con el temporal de calor, frío y lluvias

Fuente: elaboración propia con base en entrevistas realizadas en campo. 
mentación consumiendo más frutas y verduras, mantenerse hidratados y procurar vestimenta adecuada que les mantenga frescos:

La segunda parte de la biblia dice que supuestamente se va a acabar el mundo en fuego y le dije a mi mamá, pues ya se está acabando. Ella hasta dice, "si esto es el infierno ya me voy a portar bien". Y no, ¡lo que te falta!, yo no puedo dormir del calorón. Te digo que sí es cierto, todo el mundo se queja del calor porque sí está más cañón (intenso) y todo el año. Ya no hay fresco más que cuando está nubladito y dices que rico y hasta aprovechas, pero es muy raro. Cual salir a caminar ya, si son las siete de la noche y es un calorón. Yo que tengo mi bebé de un año, cuando llegue a los quince, ¿cómo va a vivir?, porque a mí ya me va a estar llevando la fregada, me va a faltar poquito, pero él va a empezar. Nos estamos acabando nuestro ecosistema y al rato va a ser un problemón. (Entrevistada 13, julio 5, 2017)

\section{Peligros en la comunidad: experiencias con inundaciones}

En términos de los peligros asociados al cambio climático, en los últimos quince años, los entrevistados refirieron que las inundaciones son el evento más frecuente y peligroso en su comunidad (78\%). En seguida destacan los incendios forestales al mismo nivel que el calor extremo (52\%), en tercer lugar, el deslizamiento de tierras desde los cerros y la contaminación del aire, ambos en el mismo lugar (14\%) y, finalmente, el frío extremo (11\%). Los entrevistados que señalaron las inundaciones como el principal evento de peligro en la comunidad han vivido una experiencia personal frente a dicho riesgo. Aunado a esto, como lo mostró la tabla 2, los participantes expresaron que el temporal de lluvias ha cambiado y ahora hay un menor número de días lluviosos pero mayor número de días con lluvia intensa.

Las inundaciones han implicado una serie de experiencias importantes relacionadas a pérdidas y afectaciones en la zona, especialmente sobre las personas que viven cerca los arroyos de la zona. Existe un sentimiento de temor que es consecuencia de experiencias pasadas ya que este peligro ha llegado a manifestarse con la magnitud suficiente para derribar viviendas, arrastrar vehículos, acabar con el patrimonio de familias e incluso provocar víctimas mortales al ser llevadas por las aguas de los arroyos. Además, las inundaciones son un evento que sucede cada año en El Colli:

Por aquí donde yo vivo, más bien, está un arroyo y cuando llueve bastante se lleva todo lo que hay de paso. Cada año en las lluvias hay muerto. (Entrevistado 14, julio 3, 2017)

Por ahí más o menos, en donde está aquel árbol, ahí fue a parar la señora que se la trajo el agua. Pobrecita, se murió, los niños quedaron huérfanos. La señora fue a dar hasta acá. Ya le digo, se quedó allá la pobrecita, se quedó allá muerta. Hay cosas que pues ni modo, hay cosas que pasan 
así, pobrecita gente. Se murió esa señora, se la trajo el agua porque salvó a sus hijos, pero no se pudo salvar ella, pobrecita. Hay muchas cosas que pasan, pero nadie nos atiende. (Entrevistada 4, julio 17, 2017)

Es posible identificar un sentimiento de impotencia y resignación por parte de los entrevistados al verse inmersos en esta situación que consideran sin remedio. Según las referencias obtenidas en las entrevistas, los bajos ingresos han orillado a los pobladores a vivir en sitios con mayor exposición al riesgo de inundación. Al mismo tiempo, esta condición financiera les origina mayor sensibilidad y menor posibilidad de solventar los gastos que derivan de los daños:

No, aquí me llegó el agua como a metro y medio de la pared, yo me salí por una ventana y ya me daba el agua aquí. En realidad, aquí es un peligro, ¿sabes lo que nos decían?, que para qué compramos en zonas de riesgo, echándonos la culpa a nosotros, le dijo una señora, "mire, compramos porque en realidad nuestro recurso no nos alcanzó, por eso compramos ahí". Yo hubiera comprado en Bugambilias (una zona habitacional de lujo en el municipio de Zapopan), ¿quién quiere vivir aquí? (Entrevistado 17, julio 7, 2017)

Además de que esta es una zona pobre, con trabajos compran sus muebles, imagínate, cada año se les echan a perder, cobijas, camas, de lo poquito que tiene. Sí da coraje. (Entrevistado 18, agosto 11, 2017)

Frente a esto, los entrevistados se pronunciaron con hartazgo ante las diversas problemáticas que suscitan en su entorno, sobre todo al no ver acciones claras por parte de las autoridades responsables, comenzando por

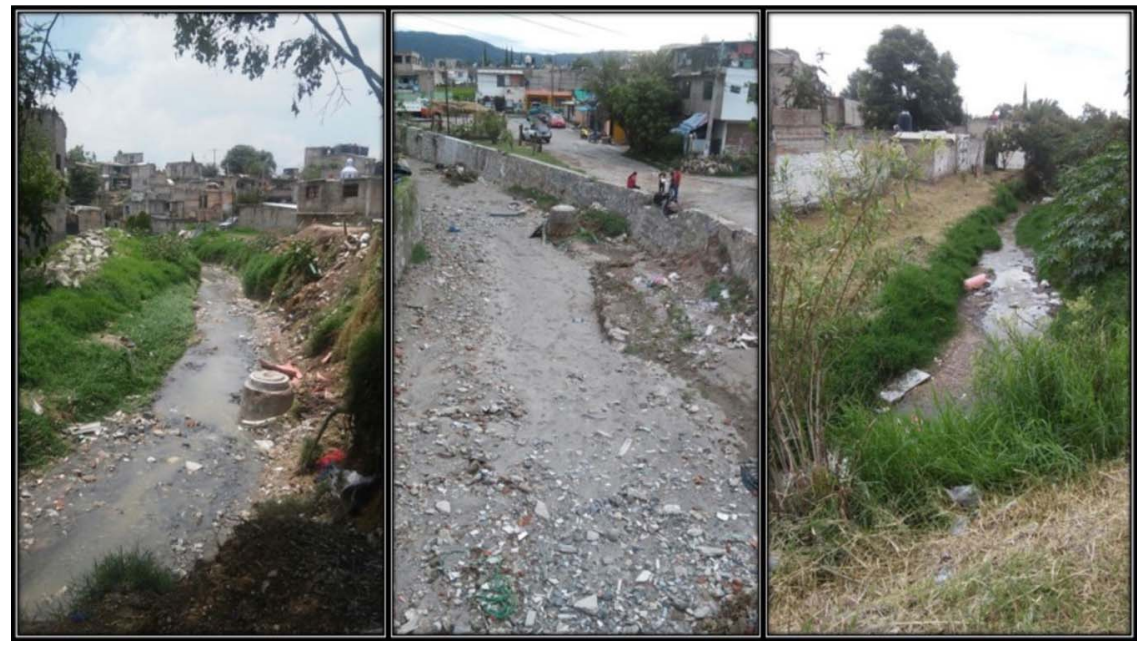

Fotografía 3 - Arroyos urbanizados en la colonia Arenales Tapatíos

Fuente: propia. 
el ayuntamiento de Zapopan en término, por ejemplo, de la provisión de servicios básicos y el mejoramiento de los espacios públicos. Esto genera un sentimiento de comunidad olvidada o excluida. Las personas entrevistadas que han tenido una experiencia más cercana a las inundaciones son quienes dan mayor énfasis a la insuficiencia de apoyo gubernamental. Esto ha llevado a que las personas tomen medidas en sus propias viviendas como colocar algunos muebles sobre ladrillos para situarlos a mayor altura y construir una especie de barrera con costales de arena para evitar el ingreso de agua a sus hogares:

\begin{abstract}
Yo veo que la gente es la que hace sus cosas para prevenir por si vuelve a pasar algo. Subieron sus banquetas, taparon puertas, abrieron otras puertas, hicieron cuartos altos en caso de eso (inundaciones). Subir cosas y protegerse ellos. Pero que yo sepa que el gobierno viene aquí, no. (Entrevistado 10 , julio 5,2017 )
\end{abstract}

Durante el diseño de la entrevista no contemplamos abarcar el tema de la inseguridad que suele prevalecer en zonas de pobreza urbana, sin embargo, en el caso de El Colli, todos los entrevistados comentaron haber tenido una experiencia cercana de robo, venta y consumo de drogas, peleas entre pandillas e incluso asesinatos, entre otros. La situación de inseguridad es tan pronunciada que, en experiencia de los entrevistados, las patrullas de policía tienen miedo de hacer su trabajo. Lo anterior también ha incrementado la desconfianza hacia las instituciones públicas, incrementando el sentimiento de comunidad olvidada que muchas personas expresaron. Esto ha traído efectos negativos en la cohesión social haciendo que algunos entrevistados comentaran que las personas de El Colli no quieren cooperar para exigir colectivamente mejorías para su comunidad:
Allá donde vivo (hacia las faldas del bosque La Primavera) a veces hay pleitos de borrachos, tiran balazos y todo eso. Esta muy feo aquí. Hablas a las patrullas y no vienen. Tienen miedo. Porque sí saben en dónde está el peligro, pero no quieren venir. Les da miedo. De tontos se van a arries- gar. (Entrevistado 8, junio 3, 2017)

Finalmente, podría pensarse que el cambio climático no tendría lugar en la percepción o conocimiento de las personas. Sin embargo, parte importante de los participantes expresaron preocupación al hablar de cambio climático a pesar de no conocer claramente a qué se refiere el término. En este sentido las evidencias aportadas a través de las entrevistas permiten reconocer que el tema de cambio climático sí resulta importante para los participantes y por tanto está presente en contextos de pobreza urbana, lo cual da pie a la posibilidad de intervenir con mayor facilidad en la co- 
munidad a través de espacios que permitan trabajar la comunicación del riesgo y la gestión de este:

Pues sí preocupa porque, ahorita tantos incendios, y tanta cosa que ha habido, ¿mañana qué va a pasar? Todo va cambiando. Ahorita ya no sabes ni de qué cuidarte. (Entrevistada 14, julio 3, 2017)

¡Aaay claro, claro! La gente que, qué le vamos a dejar a los nietos, a la futura familia qué le vamos a dejar, imagínate. Claro que me preocupa (el cambio climático). Es lo más, ahorita yo pienso que es lo más preocupante. (Entrevistada 11, julio 7, 2017)

\section{Discusión}

Los resultados del estudio nos permiten encontrar aspectos clave que expresan la percepción sobre los riesgos asociados al cambio climático tanto para la salud y el bienestar de la población en un contexto de pobreza urbana. La mayor cantidad de problemas en salud (sean enfermedades concretas o síntomas) percibidos por los habitantes de las colonias Miramar, Arenales Tapatíos y Lomas de La Primavera, están asociadas con las altas temperaturas, lo cual es importante en términos del incremento generalizado de días cálidos durante el año en el AMG (Curiel, 2015). La variabilidad climática, entendida como cambios abruptos de temperatura durante periodos de tiempo muy cortos o incluso durante el transcurso del día, fue calificada de impredecible y a esto se le atribuye el principal riesgo para la salud. Al mismo tiempo la principal amenaza para la vida y el patrimonio de las personas son las inundaciones. Además, los participantes perciben un aumento de lluvias intensas en el área de estudio, lo cual propicia una sensación mayor riesgo con el paso del tiempo según la experiencia de las personas.

Los impactos directos e indirectos del cambio climático en la salud han propiciado el desarrollo de métodos epidemiológicos para generar datos empíricos al respecto, es decir, métodos encaminados a describir y explicar la dinámica de la salud de la población (Scovronick et al., 2015). Sin embargo, para el caso de estudio aquí presentado, los datos capturados y reportados por las instituciones de salud son insuficientes para obtener resultados confiables respecto a las causas y efectos en salud asociadas al cambio climático. Esta ausencia de datos se debe en parte a que, al ser asentamientos irregulares, no son considerados dentro de los servicios básicos ni de las colonias que forman parte de los datos estadísticos de población. Frente a este reto, destacan algunos estudios que han optado por acercamientos de carácter cualitativo y comunitario para 
generar una primera aproximación a la relación entre salud y cambio climático en contextos de desventaja (Singer, 2016). Estos estudios identifican que las voces de poblaciones consideradas vulnerables son clave para la toma de decisiones y acciones frente al cambio climático por parte de las organizaciones gubernamentales, pues son ellos quienes conocen mejor su realidad. Este tipo de aseveraciones que sostienen la importancia de la percepción del riesgo en términos comunitarios se encuentran bien sustentadas en estudios que abordan otros problemas de salud con una relación más directa a situaciones de degradación ambiental (Auyero \& Swistun, 2007).

La escasez de recursos fue un factor importante que propició que los entrevistados decidieran vivir en espacios que carecen de servicios urbanos básicos y que están mayormente expuestos a la ocurrencia de inundaciones debido a su cercanía con antiguos arroyos del bosque La Primavera. Algunos autores han encontrado que la pobreza suele orillar a las personas a vivir en estos espacios ya que los precios tienden a ser menores cuando existen riesgos ambientales (Qin et al., 2015). Esta dinámica es característica del crecimiento urbano hacia las afueras de la periferia del AMG es característico de los municipios de Zapopan y Tlajomulco, propiciando espacios de marginación y pobreza. Esta situación ha originado un contexto de marginación urbana que propicia un estado de vulnerabilidad frente a peligros como las inundaciones, lo cual implica un riesgo para la salud y a la propia vida de las personas.

El proceso de generación de espacios urbanos marginales ubicados en zona de riesgos ambientales asociados al cambio climático como las inundaciones ha sido identificado por otros autores (Hallegatte et al., 2015; Kawasaki et al., 2020). Esta situación nos habla de la vulnerabilidad como un factor socialmente producido. Esta investigación aborda la vulnerabilidad en el sentido que expone García (2018), como las condiciones materiales de existencia que ponen en peligro al ser vivo, familia, grupo social o sociedad, las cuales pueden provenir del contexto o del entorno, así como de los recursos o activos con los que se cuente. Lo anterior es importante en términos de cambio climático, pues invita a cuestionarse sobre las acciones de mitigación y adaptación tradicionales. Estas están estrechamente encaminadas a la reducción de emisiones de gases de efecto invernadero pero, dadas las condiciones de casos como el aquí expuesto, es necesario repensar la acción climática y completar la reducción de emisiones de efecto invernadero con la reducción de condiciones concretas que afectan el bienestar de las personas, como la falta de servicios básicos, la precariedad de la vivienda, el abandono de los espacios públicos, entre otros factores que propicien mejores condiciones del entorno habitado. Esto implicaría releer el cambio climático desde la literatura académica 
sobre vulnerabilidad social orientada a los desastres y riesgos ambientales (Briones, 2012).

El riesgo atribuible a las inundaciones es alto y existe un sentimiento arraigado de temor debido a la incertidumbre sobre la magnitud que podría presentarse un evento de inundación. Aun así, a pesar de que el riesgo es reconocido y esto debería llevar a una búsqueda por proteger la salud y el bienestar, el riesgo aceptable es alto. Este principio de reconocimiento y aceptación del riesgo se relaciona con el estado de pobreza pues los pobladores han adoptado la actitud de "no hay de otra", es decir, los pobladores no reconocen la posibilidad de cambiar su situación, generando un sentimiento de impotencia. Al igual que en otros estudios (Hardoy et al., 2019), esto radica en una falta de integración de las poblaciones vulnerables en la planeación y la toma de decisiones para mejorar las condiciones de su entorno. Lo anterior sustenta la importancia de avanzar en procesos participativos de investigación con la intención de mejorar la calidad de vida de las comunidades.

Finalmente, en los resultados de las entrevistas destacó la situación de inseguridad que se vive en la comunidad, pues todos los entrevistados expresaron haber tenido experiencias cercanas con este hecho. Observamos que esto incrementa la desconfianza hacia el gobierno en general debido a la percepción de una nula actuación de los cuerpos policiales, pero también genera fragmentación o debilitamiento entre los propios vecinos, ya que las personas no se sienten con la libertad de confiar entre ellas mismas. A pesar de que las personas adoptan ciertas estrategias para proteger su salud y bienestar frente a la variabilidad climática y los riesgos de inundación, la fragmentación y la inseguridad percibida son factores que debilitan las acciones colaborativas de reducción comunitaria de los riesgos.

\section{Conclusión}

A partir del análisis de los datos recabados es posible dar respuesta a nuestra pregunta general de investigación la cual se enfocó en conocer la percepción del riesgo a la salud y el bienestar frente al cambio climático en un contexto de pobreza urbana. En este sentido es posible concluir que, las percepciones del riesgo a la salud asociadas al cambio climático de los habitantes de la comunidad de El Colli tienen como origen la construcción de viviendas en un asentamiento irregular que por sus características geográficas ya es riesgoso. Esta primera condición de riesgo está directamente relacionada a la condición de pobreza, dado que al no tener alternativa de compra de casa aceptan construir de manera libre sin analizar las consecuencias posibles. 
Posteriormente la percepción del riesgo emerge a partir de los años de habitar el lugar y los eventos sucedidos, tal es el caso de las referencias en torno al cambio en el comportamiento del clima, que van desde días más calurosos y eventos de lluvias más intensas hasta la relación con problemas de salud como los dolores de cabeza, diarrea, agotamiento, estrés, entre otros. Esto nos habla del cambio climático a partir de cambios graduales que ocurren a través del tiempo, con impactos discretos, pero que repercuten paulatinamente en la salud de las personas. Estos cambios graduales en el comportamiento del clima interactúan con el contexto de la comunidad y las condiciones de vulnerabilidad se convierten en una especie de filtro para lograr el bienestar frente al cambio climático.

Esta investigación afirma que los impactos del cambio climático requieren pensarse desde las opiniones y experiencias de la gente. Esto permite reconocer que los impactos regionales y a escala locales del cambio climático están inmersos en una dinámica de vulnerabilidad desigual entre la población. De tal modo, las realidades regionales a escala local enfrentan impactos específicos en contextos particulares de vulnerabilidad frente al cambio climático. Este punto requiere incorporarse a la planeación y a la toma de decisiones en torno a la acción climática, tanto en adaptación como en mitigación. La percepción del riesgo a la salud permite posicionar el conocimiento y las experiencias cotidianas de las personas como elementos que suman a los análisis técnicos en términos, por ejemplo, de escenarios de cambio climático. Incluso podríamos pensar en escenarios sociales de cambio climático en donde el conocimiento se construye en colaboración con los actores que están directa e indirectamente involucrados, para así tomar decisiones y adoptar políticas de impacto y cambio social. El cambio climático no podrá enfrentarse desde un enfoque en donde los expertos acudan a las poblaciones a estimar el riesgo sin tomar en cuenta las experiencias, preocupaciones y saberes de los habitantes. Es necesario un abordaje que revele las particularidades experimentadas por las personas que viven directamente los problemas ambientales, un abordaje desde y para la gente, tal como lo intentamos plasmar en esta investigación.

JUAN ALBERTO GRAN CASTRO es estudiante de doctorado en ciencias sociales en el Centro de Investigación y Estudios Superiores en Antropología Social, unidad occidente. Sus temas de investigación se enfocan a los problemas ambientales relacionados con el cambio climático, especialmente en los temas de riesgo y vulnerabilidad en contextos urbanos. Email: juangran91@gmail.com

SILVIA LIZETTE RAMOS DE ROBLES es doctora en didáctica de las ciencias experimentales por la Universidad Autónoma de Barcelona. Actualmente es profesora e investigadora del Departamento de Ciencias Ambientales de la Universidad de 
Guadalajara y coordinadora de la Maestría en Ciencias de la Salud Ambiental. Sus investigaciones recurren al uso de la perspectiva sociocultural para analizar procesos de educación en ciencias. Adicionalmente sus investigaciones se focalizan en los aspectos socioambientales relacionados con la salud ambiental y la educación para la salud. Email: lizette.ramos@academicos.udg.mx

\section{NOTES}

1. El rango de pobreza corresponde al porcentaje de población que vive en condiciones de pobreza dentro de un espacio específico: muy bajo indica que entre $0 \%$ y $18 \%$ de la población total dentro de dicho espacio vive en pobreza, bajo corresponde entre $18 \%$ a $34 \%$, medio $34 \%$ a $50 \%$, alto $50 \%$ a $70 \%$ y muy alto $70 \%$ a $100 \%$ (CONEVAL, 2015).

2. El área de geoestadística básica es una escala delimitada por el Instituto Nacional de Estadística y Geografía (INEGI) para la sistematización de datos en contextos urbanos.

\section{REFERENCIAS}

Akerlof, K., DeBono, R., Berry, P., Leiserowitz, A., Roser-Renouf, C., Clarke, K. L., ... \& Maibach, E. W. (2010). Public perceptions of climate change as a human health risk: Surveys of the United States, Canada and Malta. International Journal of Environmental Research and Public Health 7(6), 2559-2606. doi:10.3390/ ijerph7062559.

Akerlof, K. L., Delamater, P. L., Boules, C. R., Upperman, C. R., \& Mitchell, C. S. (2015). Vulnerable populations perceive their health as at risk from climate change. International Journal of Environmental Research and Public Health 12(12), 15419-15433. doi:10.3390/ijerph121214994.

Auyero, J., \& Swistun, D. (2007). Expuestos y confundidos: un relato etnográfico sobre sufrimiento ambiental. Iconos. Revista de Ciencias Sociales 28, 137-152.

Beck, U. (1999). La sociedad del riesgo. Hacia una nueva modernidad. Barcelona: Paidós.

Beck, U. (2010). Remapping social inequalities in an age of climate change: for a cosmopolitan renewal of sociology, Global Networks, 10(2), 165-181.

Bolívar Botía, A. (2002).¿“De nobis ipsis silemus"?: Epistemología de la investigación biográfico-narrativa en educación. Revista electrónica de investigación educativa 4(1), 1-26.

Briones, F. (2012). Perspectivas de investigación y acción frente al cambio climático en Latinoamérica. Venezuela: LA RED.

Chávez-Rodríguez, L. (2016). La importancia de la interseccionalidad en la vulnerabilidad social ante eventos hidrometeorológicos extremos en Yucatán, México. En G, Velázquez, G. Vázquez, Z. De Luca \& C. Sosa (Coords.), Transformaciones ambientales e igualdad de género en América Latina: temas emergentes, estrategias y acciones (pp. 19-42). México: UNAM. 
CONEVAL - Consejo Nacional de Evaluación de la Política de Desarrollo Social. (2015). Pobreza urbana en México. Descargado de https://www.coneval.org .mx/Medicion/Paginas/POBREZA-URBANA-EN-MEXICO-2015.aspx.

CONEVAL - Consejo Nacional de Evaluación de la Política de Desarrollo Social (2018). Medición de la pobreza. Descargado de https://www.coneval.org.mx/ Medicion/MP/Paginas/Pobreza-2018.aspx.

Curiel, A. (2015). El clima cambiante: conocimientos para la adaptación en Jalisco. Guadalajara, México: Universidad de Guadalajara.

De la Peña, G. (2000). El Colli: surgimiento y contracciones de un asentamiento irregular. En Ayuntamiento de Zapopan (Comp.), Lecturas Zapopanas (pp. 36-56). Jalisco, México: El Colegio de Jalisco.

Del Castillo, A. (2018). Invasión de La Primavera en Santa Ana, fuera de control. Milenio. Guadalajara, Jalisco, 16 julio. Descargado de https://www.milenio .com/politica/comunidad/invasion-de-la-primavera-en-santa-ana-fuera-decontrol.

Delgado, G. C. (2017). Climate change-sensitive cities: Building capacities for urban resilience, sustai-nability, and equity. Ciudad de México: UNAM.

Ferrer, R. A., \& Klein, W. M. (2015). Risk perceptions and health behavior. Current Opinion in Psychology 5(October), 85-89.

García, A. V. (2005). El riesgo como construcción social y la construcción social de riesgos. Desacatos 19(sep./dic.), 11-24. doi.org/10.29340/19.1042.

García, A. V. (2018). Vulnerabilidad y desastre: génesis y alcances de una visión alternativa. En M. González de la Rocha \& G. A. Savarí, Pobreza y vulnerabilidad: debates y estudios contemporáneos en México (pp. 212-239). Ciudad de México: CIESAS.

Gaube, S., Lermer, E., \& Fischer, P. (2019). The concept of risk perception in health-related behavior theory and behavior change. En R. Martina, B. Streicher \& E. Lermer (Eds.). Perceived safety. A multidisciplinary perspective (pp. 101-118). New York: Springer.

Gran, J. A., \& Ramos De Robles, S. L. (2019). Climate change and flood risk: Vulnerability assessment in an urban poor community in Mexico. Environment and urbanization 31(1), 75-92. doi.org/10.1177/0956247819827850.

Hallegatte, S., Fay, M., Bangalore, M., Kane, T., \& Bonzanigo, L. (2015). Shock waves: Managing the impacts of climate change on poverty. World Bank Publications.

Hardoy, J., Gencer, E., \& Winograd, M. (2019). Participatory planning for climate resilient and inclusive urban development in Dosquebradas, Santa Ana and Santa Tomé. Environment and Urbanization 31(1), 33-52.

Intergovernmental Panel on Climate Change. (2019). Special report on the ocean and cryosphere in a changing climate. Suiza: IPCC.

Kawasaki, A., Kawamura, G., \& Zin, W. W. (2020). A local level relationship between floods and poverty: A case in Myanmar. International Journal of Disaster Risk Reduction 42(9), 101348. https://doi.org/10.1016/j.ijdrr.2019.101348.

Lezama, J. L. (2012). Riesgo Ambiental: de la ideología a su construcción social, Quid 16. Revista del Área de Estudios Urbanos, (1), 40-53. 
Liu, T., Xu, Y. J., Zhang, Y. H., Yan, Q. H., Song, X. L., Xie, H. Y., . . \& \& Ma, W. J. (2013). Associations between risk perception, spontaneous adaptation behavior to heat waves and heatstroke in Guangdong province, China, BMC public health, 13(1), 1-14.

López, N. F. (2002). El análisis de contenido como método de investigación. Revista de educación 21, 167-179.

Luhmann, N. (2006). Sociología del riesgo. México: Universidad Iberoamericana.

O'Brien, K., Eriksen, S. E., Schjolden, A., \& Nygaard, L. P. (2004). What's in a word? Conflicting interpretations of vulnerability in climate change research. CICERO Working Paper.

Oliver-Smith, A., Alcántara-Ayala, I., Burton, I., \& Lavell, A. (2017). The social construction of disaster risk: Seeking root causes. International Journal of Disaster Risk Reduction 22(November), 469-474.

OMM-Organización Meteorológica Mundial (2011). Guía de prácticas metodológicas. Suiza: OMM.

Qin, H., Romero-Lankao, P., Hardoy, J., \& Rosas-Huerta, A. (2015). Household responses to climate-related hazards in four Latin American cities: A conceptual framework and exploratory analysis. Urban Climate 14(1), 94-110. http://dx .doi.org/10.1016/j.uclim.2015.05.003.

Scovronick, N., Lloyd, S. J., \& Kovats, R. S. (2015). Climate and health in informal urban settlements. Environment and Urbanization 27(2), 657-678. doi.org/ 10.1177/0956247815596502.

Singer, M. (Ed.). (2016). A companion to the anthropology of environmental health. Hoboken, NJ: Jhon Wiley \& Sons, Inc.

Sun, L., \& Faas, A. J. (2018). Social production of disasters and disaster social constructs: Disaster prevention and management. An International Journal 27(5), 623-635. doi: 10.1108/DPM-05-2018-0135.

Watts, N., Adger, W. N., Ayeb-Karlsson, S., Bai, Y., Byass, P., Campbell-Lendrum, D., . . \& \& Depoux, A. (2017). The Lancet Countdown: Tracking progress on health and climate change. The Lancet 389(10074), 1151-1164. https://doi .org/10.1016/S0140-6736(16)32124-9.

\section{Perceptions of health risks due to climate change in Mexico}

\section{Juan Alberto Gran Castro, Silvia Lizette Ramos de Robles}

Abstract: The objective of this article is to analyze the perceptions of those who live in a context of marginalization and urban poverty as to their awareness of any risks to health and well-being associated with climate change. The methodological framework for this study was qualitative and ethnographic. The main sources of information were semi-structured interviews with residents of El Colli, located in Zapopan, Jalisco, Mexico who are parents. We conclude that there are climate change-induced environmental regional particularities that result in unequal conditions 
of vulnerability and a greater occurrence of risk events for populations in marginalized and poor areas. Thus, we argue that the theoretical and methodological references that consider local knowledge are key to enabling adaptation measures aimed at social welfare.

Keywords: floods, urban poverty, urbanization, vulnerability

\section{Perception des risques sanitaires face au changement climatique au Mexique}

\section{Juan Alberto Gran Castro, Silvia Lizette Ramos de Robles}

Résumé: L'objectif de cet article est d'analyser la perception des risques pour la santé et le bien-être liés au changement climatique à partir des expériences quotidiennes de vie dans un contexte de marginalisation et de pauvreté urbaine. La méthodologie adoptée s'appuie sur une cohorte qualitative et ethnographique, dont la principale source sont des entretiens semi-structurés avec des parents résidant à El Colli, situé à Zapopan, au Jalisco, Mexique. Nous concluons que le changement climatique a des répercussions spécifiques en fonction des particularités régionales à l'échelle locale, notamment des conditions inégales de vulnérabilité de la population et de la survenue d'événements à risque. Ainsi, nous soutenons que les références théoriques et méthodologiques qui prennent en compte les connaissances locales sont essentielles pour promouvoir des mesures d'adaptation visant le bien-être social.

Mots clés: inondations, pauvreté urbaine, urbanisation, vulnérabilité. 